OPEN ACCESS

Edited by:

Haitao Shi,

Hainan University, China

Reviewed by:

Amit Srivastava,

Purdue University, United States

Alfredo J. Ibáñez,

Pontifical Catholic University of Peru,

Peru

${ }^{*}$ Correspondence:

Xuan Huang

xuanhuang@nwu.edu.cn

Hong-Li An

anhongli@mail.xjtu.edu.cn

tThese authors have contributed equally to this work and share first authorship

Specialty section: This article was submitted to

Plant Metabolism and

Chemodiversity,

a section of the journal

Frontiers in Plant Science

Received: 20 March 2021

Accepted: 07 May 2021

Published: 21 June 2021

Citation:

Zhu Y, Guo M-J, Song J-B, Zhang S-Y, Guo R, Hou D-R, Hao C-Y, An H-L and Huang $X$ (2021) Roles of Endogenous Melatonin in Resistance to Botrytis cinerea Infection in an Arabidopsis Model.

Front. Plant Sci. 12:683228. doi: 10.3389/fp/s.2021.683228

\section{Roles of Endogenous Melatonin in Resistance to Botrytis cinerea Infection in an Arabidopsis Model}

\author{
Ying Zhu't, Miao-Jie Guo ${ }^{1 \dagger}$, Jian-Bo Song ${ }^{1}$, Shu-Yuan Zhang', Rui Guo', Dai-Ru Hou', \\ Cheng-Ying Hao', Hong-Li An ${ }^{2 *}$ and Xuan Huang ${ }^{1 *}$
}

${ }^{1}$ Provincial Key Laboratory of Biotechnology of Shaanxi, Key Laboratory of Resource Biology and Biotechnology in Western China, Ministry of Education, College of Life Sciences, Northwest University, Xi'an, China, ${ }^{2}$ Center for Translational Medicine, The First Affiliated Hospital of Xi'an Jiaotong University, Xi'an, China

Melatonin is an important bioactive molecule in plants. Two synthetases, $\mathrm{N}$-acetylserotonin methyltransferase (ASMT) and serotonin N-acetyltransferase (SNAT) are involved in the final two steps of melatonin synthesis. Melatonin participates in responses to a variety of biotic and abiotic stresses in plants, but few studies have addressed the roles of endogenous melatonin in pathogen resistance. We investigated the role of endogenous melatonin in resistance to Botrytis cinerea infection in an Arabidopsis thaliana model system. Plant lines that overexpressed ASMT or SNAT through genetic manipulation showed upregulated expression of resistance genes $P R 1$ and $P R 5$, transcription factor gene WRKY33, and jasmonic acid (JA) defense pathway marker gene PDF1.2, and downregulated transcription factor gene MYC2 in JA signaling pathway. Higher melatonin content also enhanced the activity of antioxidant enzymes superoxide dismutase (SOD) and peroxidase (POD), increased JA content, reduced plant disease symptoms, and reduced lesion size in leaves. These findings indicate that endogenous melatonin enhances plant resistance to $B$. cinerea infection. In contrast, ASMT and SNAT gene silencing lines showed opposite results and were more susceptible to $B$. cinerea. Thus, it can be demonstrated that melatonin functions as an effective regulator of plant stress resistance at the genetic level. A schematic model is presented for its role in resistance to $B$. cinerea infection. Our findings also helped to elucidate the associated signal transduction pathways and interactions between melatonin and other plant hormones.

Keywords: melatonin, Botrytis cinerea, Arabidopsis thaliana, $\mathrm{N}$-acetylserotonin methyltransferase, serotonin $\mathrm{N}$-acetyltransferase

\section{INTRODUCTION}

Melatonin is a signaling molecule that ubiquitously exists in animal (Lerner et al., 1958) and plant (Dubbels et al., 1995) cells, and its synthesis pathways in plants have been discovered (Back et al., 2016). Serotonin N-acetyltransferase (SNAT; Kang et al., 2013) and N-acetylserotonin methyltransferase (ASMT; Park et al., 2013), as the main enzymes in the synthesis pathway, directly determine the endogenous melatonin level. The function of melatonin in plants has been widely reported (Fan et al., 2018; Sun et al., 2020). It can respond to various 
biotic (Mandal et al., 2018; Zhao et al., 2021) and abiotic (Chen et al., 2017; Yao et al., 2020) stresses to resist the influence of environmental changes during plant growth and development. The immune response of melatonin in plants requires the participation of many signaling molecules, such as reactive oxygen species (ROS; Pardo-Hernández et al., 2020) and Nitric Oxide (NO; Zhu et al., 2019), to transmit both intracellular and intercellular signals. In the field of biotic stress, melatonin is involved in plant resistance to numerous fungus (Moustafa-Farag and Almoneafy, 2019). The growth of certain plant fungi, including Alternaria spp. and Fusarium spp., is inhibited by treatment with melatonin. Increased melatonin levels in plants enhance resistance to Sphaerotheca fuliginea and oomycetes, and sensitivity to Phytophthora infestans (Zhang et al., 2017; Mandal et al., 2018). Melatonin pretreatment increases the resistance of apple (Malus prunifolia) to Marssonina apple blotch (Diplocarpon mali), by promoting expression of chitinase genes, regulating hydrogen peroxide $\left(\mathrm{H}_{2} \mathrm{O}_{2}\right)$ and pathogenesis-related proteins (PR proteins; Yin et al., 2013). In banana (Musa acuminata), melatonin treatment induces the production of defense-related plant hormones [IAA, salicylic acid (SA), JA, ethylene] by regulating the expression of MaHSP90, thereby enhancing resistance to Fusarium wilt (Wei et al., 2017).

Botrytis cinerea often termed "gray mold", is a necrotrophic pathogenic microorganism (fungus) responsible for major economic losses (related to its wide hosting range) considered second only to those of penicillin fungal pathogens (Dean et al., 2012). It kills host cells by secreting the sesquiterpene metabolite botrydial, and by producing ROS that induces oxidative outbreaks (Feng and Shan, 2014). Botrytis cinerea also degrades pectin in plant cell walls by synthesizing polygalacturonase, keratin enzyme, and cell wall degradation enzyme, thereby promoting invasion and damage to the plant (Liu et al., 2017). Local immune responses of plants to B. cinerea infection result in integration and expression of PR proteins, steady-state regulation of plant hormones, ROS production, and accumulation of secondary metabolites such as JA (Pieterse et al., 2009).

Plant hormone signal transduction is an important component of the local immune response, and JA in particular plays a crucial role in defense against B. cinerea (AbuQamar et al., 2017). In Arabidopsis, a JA-knockout mutant (coi1) defective in the perception of JA signal shows higher susceptibility to B. cinerea (Pingping et al., 2017). Transcriptional coactivator mediator subunit 25 (Med25) promotes plant resistance to $B$. cinerea by inducing the expression of the JA-dependent defensive gene (An and Mou, 2013). The invasion of B. cinerea to plants activates the expression of a marker gene of the JA pathway (plant defensin 1.2; PDF1.2; Frey et al., 2018). Conversely, $M Y C 2$ plays a negative regulatory role in JA-mediated immunity and it interacts with JAZ [jasmonate zinc-finger inflorescence meristem (ZIM) domain] to promote JA decomposition, thereby reducing MYC2 expression following $B$. cinerea infection (Liu et al., 2019). Transcriptome analyses indicate the alteration of thousands of transcripts in the body of an amoeba host following $B$. cinerea invasion, whereby mitogen-activated protein kinases (MAPKs) undergo phosphorylation changes during transcription of downstream genes, in which key transcription factors are involved in the regulation of plant defense responses (Mulema and Denby, 2012). WRKY proteins are major components of transcription factors that play essential roles in both PAMP triggered immunity (PTI) and Effector triggered immunity (ETI) during plant resistance to $B$. cinerea infection (Jones and Dangl, 2006). WRKY proteins regulate defensive responses to biotrophic and necrotized pathogens (Chen et al., 2018). WRKY33 also participates in the regulation of its expression through a clear feed-forward mechanism in combination with its promoter in B. cinerea-infected plants (Mao et al., 2011).

We investigated the role of endogenous melatonin in resistance to $B$. cinerea infection in an Arabidopsis thaliana model. Using genetic manipulation techniques, the expression of SNAT and $A S M T$ was altered. After B. cinerea infection, we found that SNAT and ASMT overexpressed lines exhibit obvious disease resistance characteristics compared with Columbia (Col-0). On the contrary, disease-resistant ability in SNAT and ASMT mutant lines was weakened. The results clarified that melatonin can improve plant disease resistance to the stress of $B$. cinerea by regulating the expression of related genes and the content of phytohormone JA. These pieces of evidence further prove the role of melatonin in the field of biotic stress, especially for enhancing plant resistance to fungal invasion.

\section{MATERIALS AND METHODS}

\section{Plant Material and Pathogen Inoculation Procedure}

Arabidopsis thaliana ecotype Col-0 was used in this study. Plants were grown for 4 weeks at $22^{\circ} \mathrm{C}$ under long-day conditions (16 h of light $/ 8 \mathrm{~h}$ of dark) and light intensity $100 \mu \mathrm{mol} \cdot \mathrm{m}^{-2} \cdot \mathrm{s}^{-1}$.

The B. cinerea (B05.10) used in this study was cultivated on potato dextrose agar (PDA) medium (Zheng et al., 2015) in the dark for 5 days at a $28^{\circ} \mathrm{C}$ incubator to induce conidia production. Spores were collected and then suspended in $2 \%$ glucose solution to dilute to $1 \times 10^{5}$ conidia $/ \mathrm{ml}$. Leaves (3-4 per plant) were added dropwise with $10 \mu \mathrm{l}$ spore suspension, and kept in the dark for $24 \mathrm{~h}$ and then transfer to $16 \mathrm{~h}$ light $/ 8 \mathrm{~h}$ dark condition and light intensity $100 \mu \mathrm{mol} \cdot \mathrm{m}^{-2} \cdot \mathrm{s}^{-1}$.

\section{Obtain Transgenic Plants}

Using the technique of artificial miRNA (amiRNA; Schwab et al., 2006), we successfully constructed miR172-snat and miR172-asmt vectors. Then they were separately transferred into Col-0 to silence melatonin synthesis genes via the Agrobacterium-mediated transformation method. Seeds were selected by adding $50 \mathrm{mg} / \mathrm{ml} \mathrm{kan}$ and $30 \mathrm{mg} / \mathrm{ml}$ cef in $1 / 2$ MS medium and T3 generation transgenic lines (asmt-1, asmt2, snat-1, and snat-2) were used in the following experiments. Genes overexpression lines (ASMT-OE-1, ASMT-OE-2, SNAT$O E-1$, and $S N A T-O E-2)$ were constructed in our laboratory by inserting SNAT and ASMT gene complementary DNA (cDNA) into the binary vector pRI101-AN (TaKaRa, Tokyo, Japan). 


\section{Quantitative Real-Time RT-PCR Analysis}

After inoculation with $B$. cinerea strains for $48 \mathrm{~h}$, total RNA was isolated from leaves using RNeasy Plant Mini Kit (TaKaRa, Tokyo, Japan), then reverse-transcribed to cDNA with the PrimeScript ${ }^{\mathrm{TM}} \mathrm{II} 1$ st strand cDNA Synthesis kit (TaKaRa, Tokyo, Japan). The expressions of relevant genes were evaluated by quantitative real-time RT-PCR (qRT-PCR; model CFX96; Bio-Rad).

The Actin8 (AT1G49240) genes were used as internal controls. Each experiment was repeated at least three times.

\section{Determination of Activities of Antioxidant Enzymes Superoxide Dismutase and Peroxidase}

After B. cinerea treatment for 24,48 , or $72 \mathrm{~h}$, plant leaves $(0.1 \mathrm{~g})$ were ground separately in liquid nitrogen, incubated

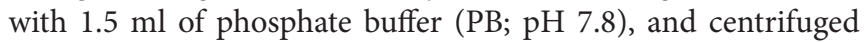
$(4,000 \mathrm{rpm}, 15 \mathrm{~min})$. The supernatant was taken as the solution to be tested.

Superoxide dismutase (SOD) and peroxidase (POD) activity was determined following Zang et al. (2015).

\section{Quantification of Melatonin and JA Content}

Plant tissues $(0.1 \mathrm{~g})$ treated by $B$. cinerea for 24,48 , or $72 \mathrm{~h}$ were grounded in liquid nitrogen and added $900 \mu \mathrm{l} 0.01 \mathrm{M}$ PBS ( $\mathrm{pH} 7.4)$, then ultrasonicated for $30 \mathrm{~min}$ and centrifuged $(12,000 \mathrm{rpm}, 10 \mathrm{~min})$ at $4^{\circ} \mathrm{C}$, The supernatants were ready to measure the content of melatonin and JA.

Melatonin content was quantified using the Melatonin Enzyme-Linked Immunosorbent Assay Kit (Jianglai, Shanghai, China), with absorbance read at $450 \mathrm{~nm}$. JA content was determined using the Plant JA ELISA KIT (Jianglai, Shanghai, China) containing anti-JA polyclonal antibodies, with absorbance read at $490 \mathrm{~nm}$. All measurements were performed in triplicate with samples collected from three biological replicates.

\section{Trypan Blue Staining}

Leaves treated by $B$. cinerea after 3 days were stained with $0.4 \%\left(\mathrm{w} / \mathrm{v}\right.$ ) Trypan Blue solution for $2 \mathrm{~h}$ at $37^{\circ} \mathrm{C}$, and chlorophyll was eliminated with $95 \%$ ethanol. The samples were observed under a dissecting microscope (Nikon C-LEDS; Nikon, Tokyo, Japan).

\section{Determination of Malondialdehyde Content}

Following B. cinerea treatment for 24,48 , or $72 \mathrm{~h}$, plant tissues $(0.1 \mathrm{~g})$ were ground in liquid nitrogen and added with $900 \mu \mathrm{l}$ of PB. After centrifugation $(4,000 \mathrm{rpm}, 10 \mathrm{~min}), 50 \mu \mathrm{l}$ of the supernatant was used as the reaction solution and assayed with an malondialdehyde (MDA) kit (Jiancheng, Nanjing, China). The absorbance of the supernatant was detected at the wavelengths of $532 \mathrm{~nm}$.

\section{Leaf Damage Area Calculation}

Leaves of plants treated with $B$. cinerea for 4 or 6 days were removed and photographed. Leaf damage area data were taken for the 6-day-treated plants and statistically analyzed using Image J $1.52 \mathrm{~s}$ software program (NIH Image, Bethesda, MD, United States).

\section{Statistical Analysis}

Analyses were performed using the Origin software program V. 8.0, with values expressed as mean \pm SE. Differences between means were evaluated by one-way ANOVA and considered significant for $p<0.05$.

\section{RESULTS}

\section{Endogenous Melatonin Alters Stress Response in B. cinerea-Infected Plants}

To evaluate the role of endogenous melatonin in response to $B$. cinerea invasion and regulation of plant defensive signaling pathways, we analyzed the expression of resistance genes $P R 1$, PR5, PDF1.2, MYC2, and WRKY33 (Figure 1). Spore suspension droplets were taken from leaves of $B$. cinerea-infected plants at 48 h. PR1 expression was significantly higher in the overexpression lines (most notably ASMT-OE-1) than in Col-0. In contrast, its expression was lower in the gene silencing lines (most notably asmt-1; Figure 1A). Expression of PR5, another important gene in defensive responses, was similarly increased in overexpression lines following $B$. cinerea infection (Figure 1B). Expression of PDF1.2, an important gene in the JA-dependent pathway, was increased 37-, 4.8-, 7-, and 2.8-fold (respectively) in SNAT-OE-2, ASMT-OE-1, ASMT-OE-2, and SNAT-OE-1 relative to Col-0. In contrast, its expression was lower in asmt-1 and snat-2 (respectively $~ 33$ and 37\% of Col-0 value), and not notably different in asmt-2 or snat-1 (Figure 1C). Expression of $M Y C 2$, a negative regulatory gene in the JA-dependent signaling pathway, showed an opposite trend. It was lower in overexpression lines (SNAT-OE-2 value $~ 17 \%$ of Col-0 value) and increased in gene silencing lines (Figure 1D). Expression of $W R K Y 33$, a transcriptional regulator gene, varied less in the modified lines than that of PR1 or PR5. The transcription level of WRKY33 was increased in the SNAToverexpression lines, particularly SNAT-OE-2, whose value was 2.86 times that of Col-0. WRKY33 expression was lower in gene silencing lines, most notably snat-1 (value $\sim 50 \%$ that of Col-0; Figure 1E).

These findings indicate that the expression of ASMT and $S N A T$ genes plays an important role in regulating the expression of PR1, PR5, PDF1.2, MYC2, and WRKY33. Downregulation of ASMT and SNAT inhibits expression of B. cinerea infection resistance genes, thereby increasing susceptibility of plants to fungal pathogens. Upregulation of ASMT and SNAT in overexpression lines enhances resistance to $B$. cinerea. In summary, plant defensive responses to $B$. cinerea infection are strongly affected by alterations in levels of the two melatonin synthesis enzymes or of endogenous melatonin. 


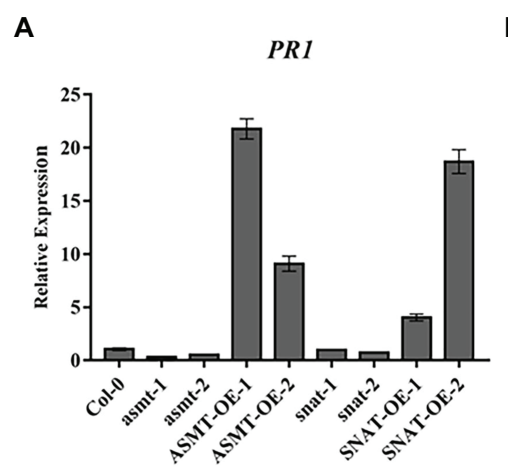

B

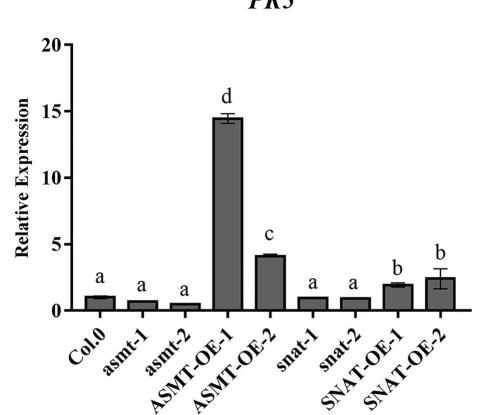

C

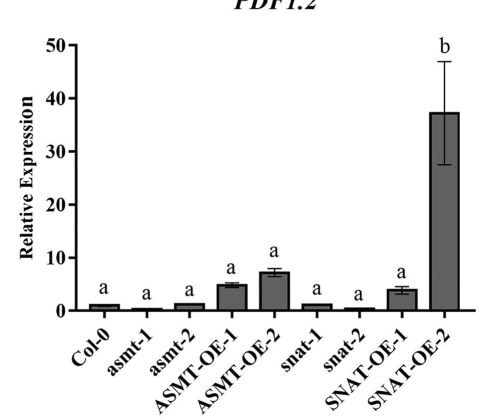

D

MYC2

E

WRKY33
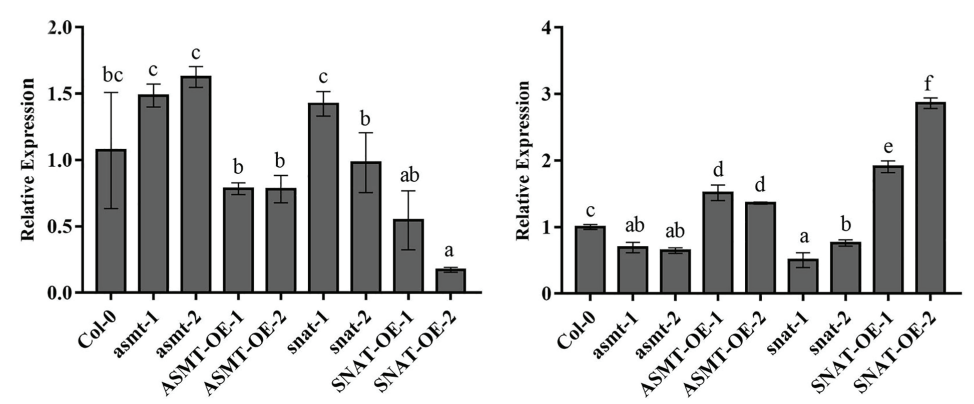

FIGURE 1 | Expression of defends signaling-related gene following Botrytis cinerea treatment. The transcript levels of PR1 (A), PR5 (B), PDF1.2 (C), MYC2 (D), and (E) WRKY33 were determined by RealTime Quantitative Polymerase Chain Reaction (RT-qPCR). RNA extracted from rosette leaves of 4-week old wild-type (Col-0), N-acetylserotonin methyltransferase (ASMT)-overexpressed (ASMT-OE-1, ASMT-OE-2) plants, serotonin N-acetyltransferase (SNAT)-overexpressed (SNATOE-1, SNAT-OE-2) plants, snat silencing lines (snat-1, snat-2), and asmt silencing lines (asmt-1, asmt-2) at $48 \mathrm{~h}$ after the B. cinerea infection. PR1 (AT2G14610), pathogenesis-related protein 1; PR5 (AT1G75040), pathogenesis-related protein 5; PDF1.2 (AT5G44420), plant defensin 1.2; MYC2 (AT1G32640); and WRKY33 (AT2G38470.1). The data (mean \pm SD) were calculated using three replicate assays, with the SEs indicated by the vertical bars. Different lowercase letters indicate statistically significant differences $(p<0.05)$.

\section{Effects of Melatonin Levels on Resistance to $B$. cinerea Infection}

To further investigate the roles of ASMT and SNAT genes in pathogen resistance, we measured endogenous melatonin levels in the overexpression and gene silencing lines. Melatonin levels were higher after $48 \mathrm{~h} \mathrm{~B}$. cinerea treatment relative to 0 or $24 \mathrm{~h}$ treatment, most notably for ASMT-OE-1 (Figure 2). The increase was significant for overexpression lines, but not for gene silencing lines or Col-0. Melatonin levels were lower in all lines at $72 \mathrm{~h}$, most notably for asmt-1. These findings indicate that endogenous melatonin content is affected by upor downregulation of ASMT and SNAT genes, suggesting a positive regulatory effect of endogenous melatonin in resistance to $B$. cinerea infection.

\section{Effects of Endogenous Melatonin on Pathogenic Processes in B. cinerea- Infected Plants}

Superoxide dismutase is resistant to oxidative damage, and plays a key role in the removal of free radicals in plants (López-Cruz et al., 2016). SOD activity in our plant lines varied depending on B. cinerea treatment time (Figure 3A). At 24 and $48 \mathrm{~h}$, SOD activities of overexpression lines were higher than that of Col-0, whereas those of gene silencing lines were significantly lower. SOD activities of the various lines were generally lower at $72 \mathrm{~h}$ than at $48 \mathrm{~h}$, but the overall trend was consistent. POD activities in the lines did not differ significantly at $0 \mathrm{~h}$, similarly to SOD activities (Figure 3B). These findings indicate that ASMT and SNAT cope with peroxide damage by alterations of endogenous melatonin level, thereby enhancing plant resistance to fungal invasion.

Spores (conidia) of B. cinerea attach to the plant surface and germinate, and the degree of leaf damage is proportional to infection time (Williamson et al., 2007; Wang et al., 2013). Botrytis cinerea treatment did not initially cause notable damage to plants, and MDA content at $0 \mathrm{~h}$ did not differ significantly among the lines, similarly to SOD activity. At longer treatment times, B. cinerea spores reproduced parasitically on leaves, leading to a greater degree of lipid peroxidation and more damage to the plants. MDA content was the highest at $72 \mathrm{~h}$ in all strains (Figure 3C). These findings indicate that upregulation of ASMT and SNAT enhances endogenous melatonin level and reduces cell membrane damage, thus increasing resistance to $B$. cinerea and relieving biotic stress, whereas downregulation of ASMT and SNAT has the opposite effect. 


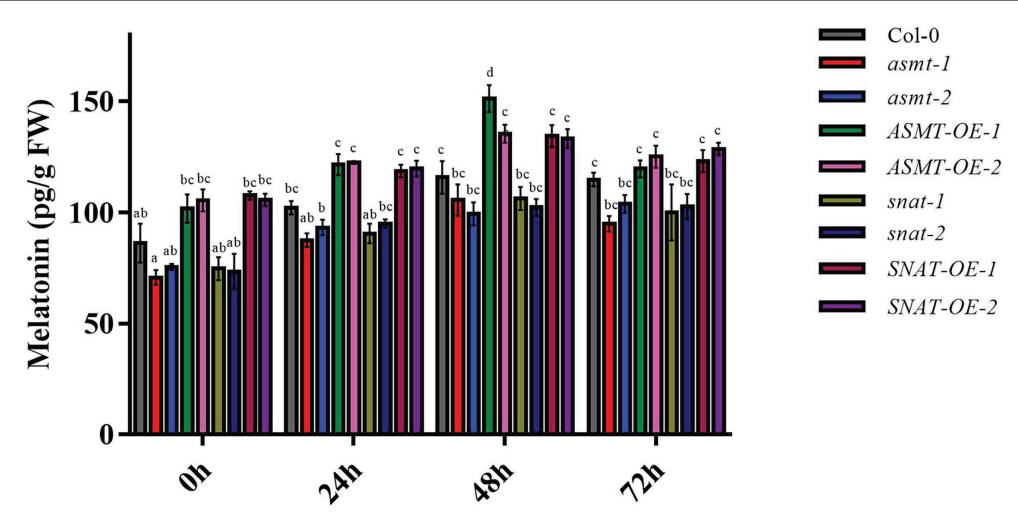

FIGURE 2 | Melatonin content following B. cinerea treatment. The content of melatonin in 4-week old wild-type (Col-0), ASMT-overexpressed (ASMT-OE-1, ASMTOE-2) plants, SNAT-overexpressed (SNAT-OE-1, SNAT-OE-2) plants, snat silencing lines (snat-1, snat-2), and asmt silencing lines (asmt-1, asmt-2) were measured at different time points $(0,24,48$, and $72 \mathrm{~h}$ ) after inoculation with $B$. cinerea. The data (mean \pm SD) were calculated using three replicate assays, with the SEs indicated by the vertical bars. Different lowercase letters indicate statistically significant differences $(p<0.05)$. FW, fresh weight.

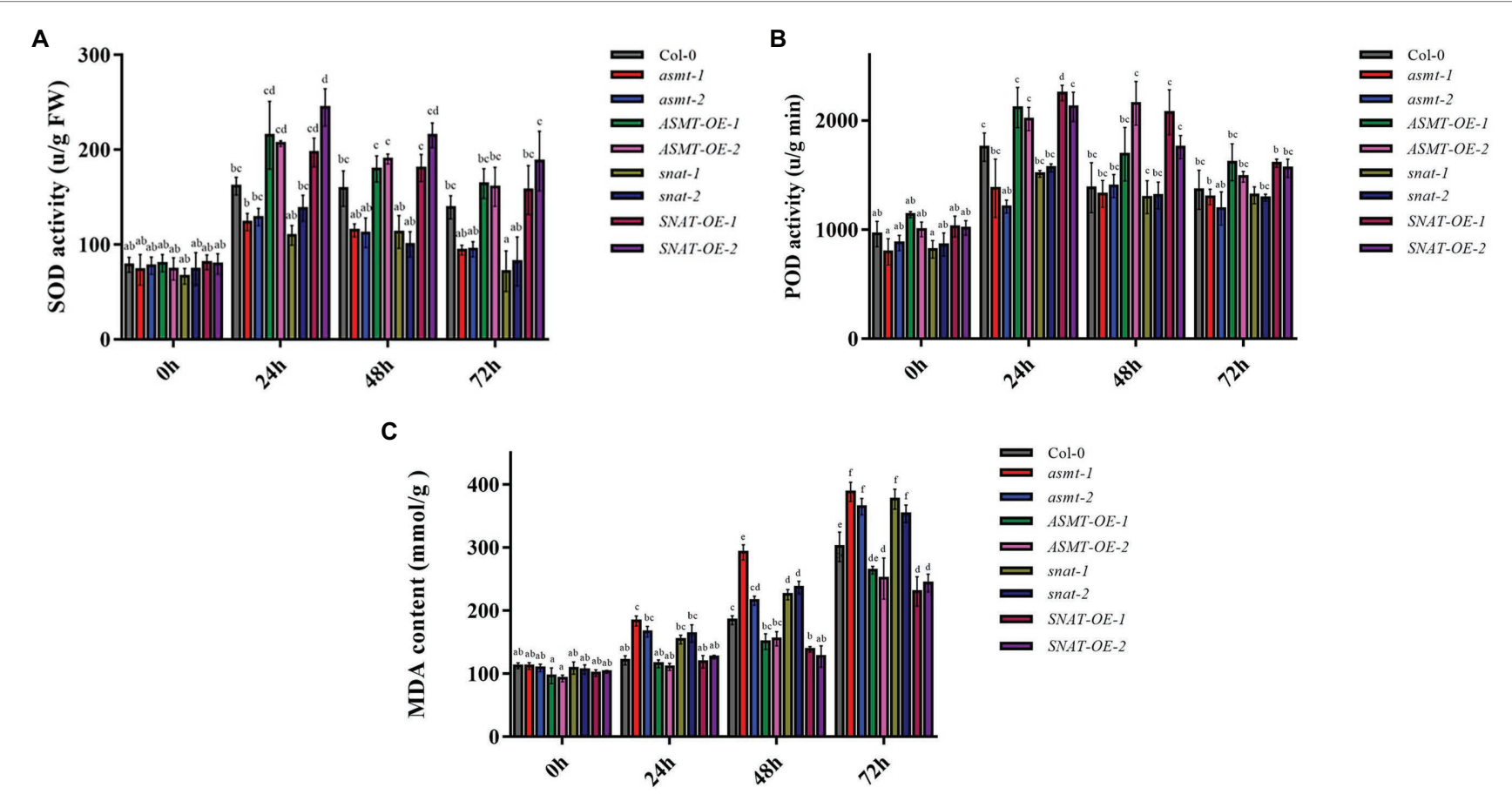

FIGURE 3 | The activities of antioxidant enzymes (A) superoxide dismutase (SOD) activity and (B) peroxidase (POD) activity and (C) the content of malondialdehyde (MDA) following B. cinerea treatment. Significant differences among 4-week old wild-type (Col-0), ASMT-overexpressed (ASMT-OE-1, ASMT-OE-2) plants, SNAT-overexpressed (SNAT-OE-1, SNAT-OE-2) plants, snat silencing lines (snat-1, snat-2), and asmt silencing lines (asmt-1, asmt-2) at different time points $(0,24,48$, and $72 \mathrm{~h})$ after the $B$. cinerea infection. The data (mean $\pm \mathrm{SD}$ ) were calculated using three replicate assays, with the SEs indicated by the vertical bars. Different lowercase letters indicate statistically significant differences $(p<0.05)$. FW, fresh weight.

\section{Effects of Leaf Damage in B. cinerea- Infected Plants}

Botrytis cinerea infection of leaves results in damage to cell membranes, such that the dye can enter cells. The degree to which cells are dyed blue reflects the extent of the disease (Ramírez et al., 2011). In Col-0, blue spots were scattered on various parts of the leaves. Blue spots on asmt-1, snat-1, asmt2 , and snat-2 leaves were deeply colored, indicating presence of more dead cells and greater susceptibility to B. cinerea. The overexpression lines showed only a few scattered dark blue spots, indicating a lesser degree of leaf cell death than in Col-0, and greater resistance to $B$. cinerea invasion (Figure 4A).

Reactions to $B$. cinerea infection varied among Col- 0 , gene silencing lines, and overexpression lines. At 4 days after inoculation (dai), gene silenced lines than in Col-0 showed greater susceptibility to $B$. cinerea more severe disease symptoms 


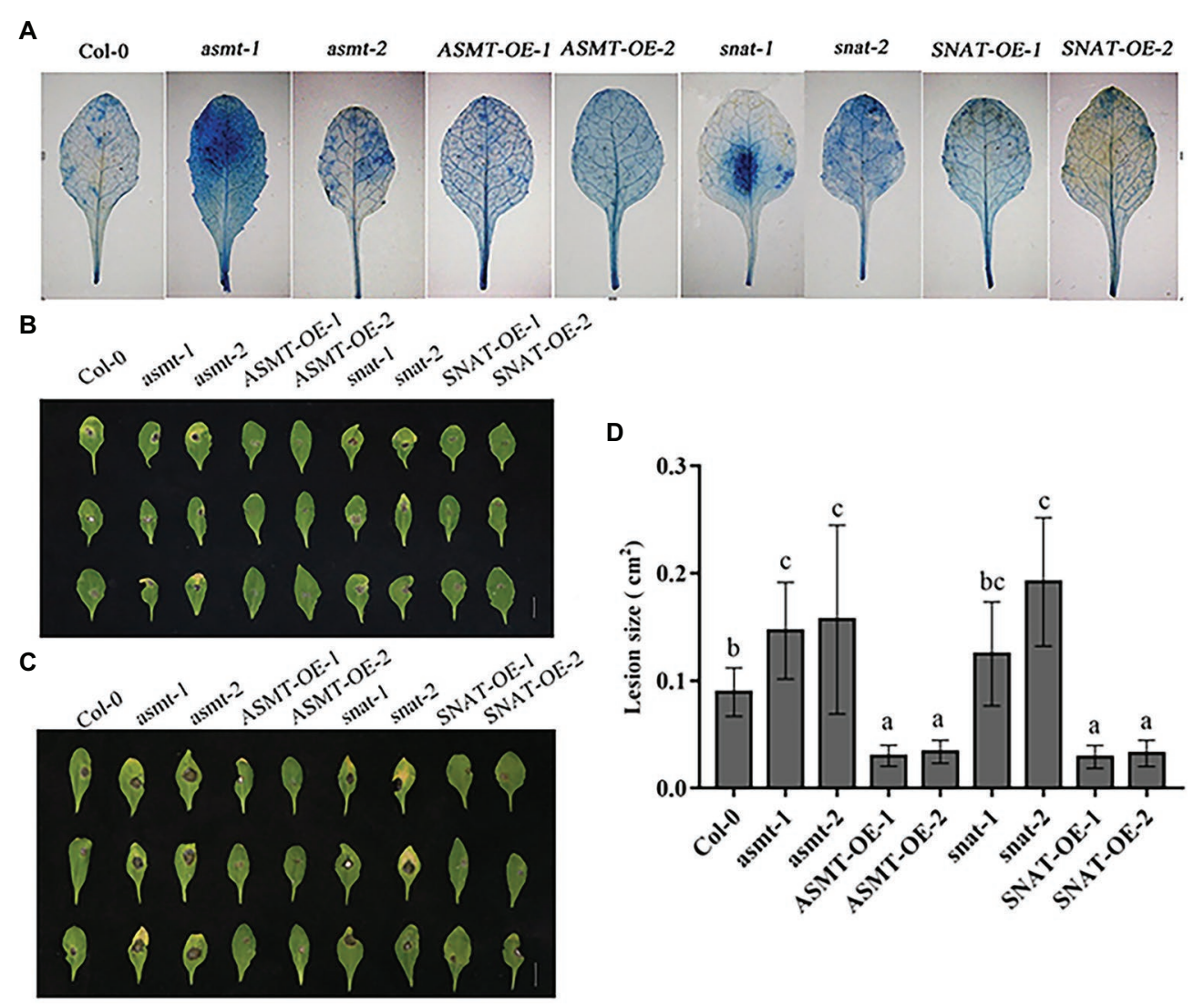

FIGURE 4 | Effects of endogenous melatonin on disease resistance to $B$. cinerea. (A) Trypan blue staining of leaves from different lines following $B$. cinerea treatment at $48 \mathrm{~h}$. (B,C) Photographs of rosette leaves cut from 4-week-old Wild-type (Col-0), ASMT-overexpressed (ASMT-OE-1, ASMT-OE-2) plants, SNAToverexpressed (SNAT-OE-1, SNAT-OE-2) plants, snat silencing lines (snat-1, snat-2), and asmt silencing lines (asmt-1, asmt-2) plants after infection with B. cinerea spores. (B) Leaves phenotype on 4 days. (C) Leaves phenotype on 6 days. (D) Lesion size. Lesion size of leaves phenotype on 6 days was measured using Image $\mathrm{J}$ software. The data (mean $\pm \mathrm{SD}$ ) were calculated using three replicate assays, with the SEs indicated by the vertical bars. Different lowercase letters indicate statistically significant differences $(p<0.05)$.

and more fungal growth. In contrast, overexpression lines showed lesser and more slowly developing disease symptoms, small disease area, and limited spore growth (Figure 4B). Leaf necrosis was more severe on 6 dai (Figure 4C) than on 4 dai (Figure 4B) for gene silencing lines, but overexpression lines showed only a minor difference between these days. These findings indicate that ASMT and SNAT gene knockdown reduces plant resistance to $B$. cinerea infection.

A graph of lesion size in the various lines (Figure 4D) also illustrates the relationship between $B$. cinerea and ASMT/SNAT. Leaf damage area for the four-gene silencing lines (most notably snat-2) was significantly larger than for Col-0. Upregulation of ASMT and SNAT promoted host defensive response.

\section{Effect of Endogenous Melatonin on JA Content}

Jasmonic acid signaling plays a key role in the $B$. cinerea infection process. We examined the effects of altered endogenous melatonin levels on JA content. At $0 \mathrm{~h}$, JA content did not differ notably among Col-0, gene silencing lines, and overexpression lines. JA content was significantly higher at $24 \mathrm{~h}$ than at $0 \mathrm{~h}$ for overexpression lines (particularly SNAT-OE-1), but such change was less notable for gene silencing lines (Figure 5). Maximal values were observed at $48 \mathrm{~h}$ for Col-0 and overexpression lines. JA content at $48 \mathrm{~h}$ was significantly higher for overexpression lines (particularly SNAT-OE-2) than for Col-0. Values at $72 \mathrm{~h}$ were lower than those at $48 \mathrm{~h}$ for Col-0 and all four overexpression lines. These findings indicate that endogenous melatonin level affects JA content for signal transduction pathways involved in pathogen resistance.

\section{CONCLUSION AND FUTURE PERSPECTIVE}

Botrytis cinerea is a necrotrophic fungus with a broad host plant spectrum (Dean et al., 2012), Plants are infected mainly by $B$. cinerea spores, which are released from previously infected plants when disturbed (Williamson et al., 2007). Some researchers have uncovered the role of melatonin in plant- $B$. cinerea 


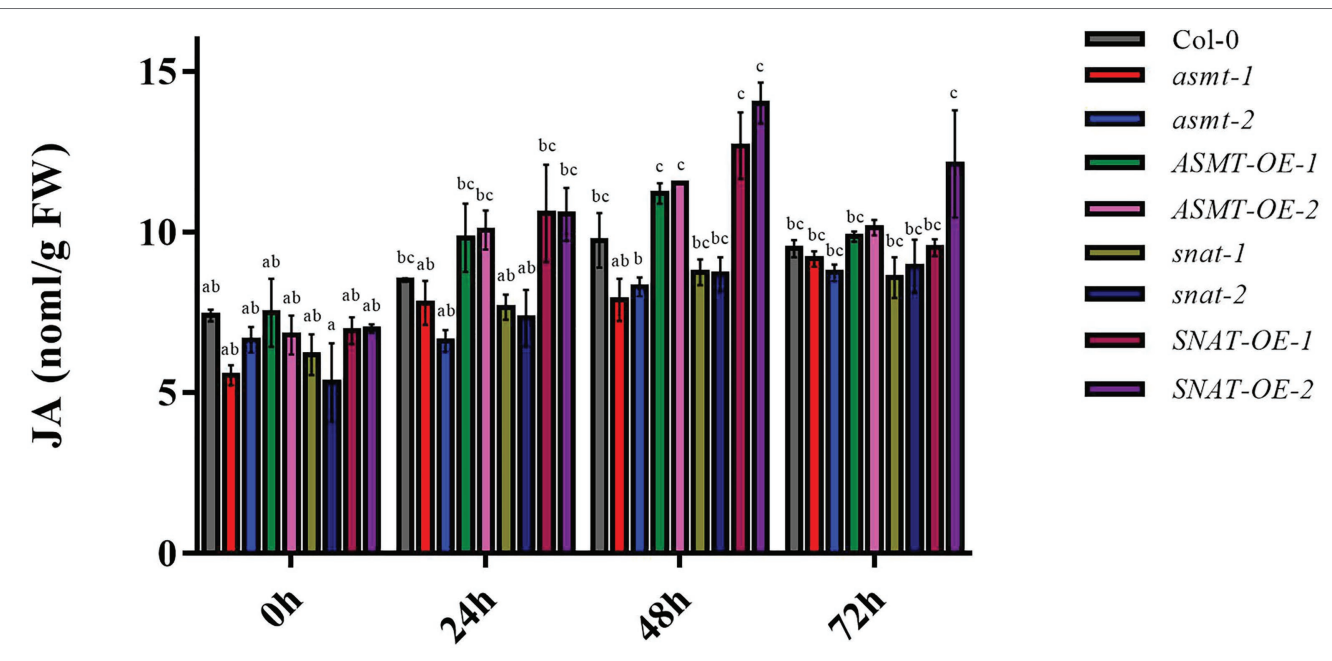

FIGURE 5 | Jasmonic acid (JA) content following B. cinerea treatment. The content of JA in 4-week-old wild-type (Col-0), ASMT-overexpressed (ASMT-OE-1, ASMT-OE-2) plants, SNAT-overexpressed (SNAT-OE-1, SNAT-OE-2) plants, snat silencing lines (snat-1, snat-2), and asmt silencing lines (asmt-1, asmt-2) plants at different time points $(0,24,48$, and $72 \mathrm{~h})$ after inoculation with $B$. cinerea. The data (mean $\pm \mathrm{SD}$ ) were calculated using three replicate assays, with the SEs indicated by the vertical bars. Different lowercase letters indicate statistically significant differences $(p<0.05)$. FW, fresh weight.

interaction, for instance, tomato (Liu et al., 2019), Fragaria ananassa (Aghdam and Fard, 2017). We are going to study the resistance of melatonin to $B$. cinerea or related fungal species in A. thaliana and other model plants in future work. Enhancing effects of exogenous melatonin on plant resistance to fungi have been observed in many studies (Amaral and Cipolla-Neto, 2018). Much less is known regarding the role of melatonin (particularly endogenous melatonin) in plant resistance to $B$. cinerea. We examined the effects of altered endogenous melatonin levels on such resistance.

Plant defense mechanisms against pathogens involve complex signaling networks, including the expression of related genes (Senthil-Kumar and Mysore, 2013). PR1 gene often plays a key role in plant resistance against necrotrophic pathogens (Frey et al., 2018). In this study, PR1 expression in ASMT and SNAT overexpression lines was significantly increased relative to Col-0 but was reduced in gene silencing lines (Figure 1A). These findings are consistent with previous reports that the increase of PR1 expression promotes plant defense against $B$. cinerea (Frey et al., 2018). PR1 is considered to be associated with SA; however, it shows increased expression mainly in defensive responses against biotrophic fungi and not those against necrotrophic B. cinerea. Functional roles of SA are complex, and functions of SA signals in plant resistance to $B$. cinerea remain unclear (AbuQamar et al., 2017). PR5, a gene co-expressed with $P R 1$, is also involved in signal transduction in plants (Siewers et al., 2005). We observed upregulation of PR5 in overexpression lines, similarly to $P R 1$, and downregulation in asmt-1 and asmt-2. However, PR5 expression in snat-1 and snat-2 did not differ notably from that in Col-0, possibly because PR5 is not a single-label gene for resistance to $B$. cinerea (Figure 1B).

Transcription factor WRKY33 is an essential component in the regulation of plant defensive responses to fungal infection. WRKY33 expression is induced in B. cinerea-infected plants. In our gene expression analysis, WRKY33 was upregulated in overexpression lines but downregulated in gene silencing lines (Figure 1E), consistently with the increased susceptibility to $B$. cinerea observed for WRKY33 mutants (wrky33-1, wrky33-2). In previous studies, WRKY33 has been suggested to participate in JA-dependent pathways and to play a negative regulatory role in JA-mediated defensive responses. However, we did not observe an association between WRKY33 increase and reduced JA content (Figure 5), indicating that this gene is not involved in the JA-dependent signaling pathway in this case. Recent reports show that WRKY33 induction in B. cinerea-infected plants does not require a JA signal molecule (CORONATINE INSENSITIVE1; COI1; Wang et al., 2015), which illustrates that WRKY33 is activated via a JA-independent pathway. On the other hand, WRKY33 is phosphorylated by MPK3/MPK6 to induce synthesis of the phytoalexin camalexin in $B$. cinerea infected plants (Mao et al., 2011), suggesting that WRKY33 expression in our ASMT and SNAT overexpression and gene silencing Arabidopsis lines may be related to MPK3/MPK6. We speculate melatonin can act directly on MAPKs and it further phosphorylates WRKY33.

Changes in growth and physiological processes of B. cinerea-infected plants are related to a variety of metabolic processes, including enzyme degradation and soluble sugar accumulation in cell walls, $\mathrm{pH}$ changes, and reduced production of antifungal compounds and secondary metabolites. Most of these changes are regulated by hormone signals, such as ethylene, Abscisic Acid, Jasmonic Acid and Salicylic Acid (Ingle et al., 2015). Studies based on transcriptome analysis suggest that the involvement of melatonin in plant-fungus interactions alters the expression of JA-related genes, and that melatonin can interact with JA to modulate plant defensive responses (Mandal et al., 2018). However, the role of melatonin in the JA pathway 
remains unclear. We observed high JA content (Figure 5) in our overexpression lines, but low JA content in gene silencing lines. Thus, increased melatonin level promoted JA accumulation, and consequently resistance to fungal infection. Increased JA content triggers signaling pathways downstream of JA. JAZ protein has an inhibitory effect on JA signaling pathway, and increased JA content leads to JAZ protein degradation, thereby reducing interaction between JAZ and MYC2 (Ruan et al., 2019), consistently with the reduced MYC2 transcription levels in our overexpression lines (Figure 1D). MYC2 is a regulatory factor that plays an essential role in the JA-dependent signaling pathway. It negatively regulates downstream signal genes $(O R A 59 / E R F 1)$ that activate plant defensive responses and trigger downstream expression of $P D F 1.2$, which plays a positive regulatory role in JA-dependent disease resistance (Frey et al., 2018). Consistently, PDF1.2 expression was increased strongly in overexpression line SNAT-OE-2, and also in ASMT-OE-1, ASMT-OE-2, and SNAT-OE-1, but was reduced in gene silencing lines asmt-1 and snat-2 (Figure 1C). These findings further confirm the involvement of melatonin in JA signaling pathway activation and in the enhancement of plant resistance to $B$. cinerea infection. ROS are among the earliest signaling molecules in the interaction between plants and pathogens. Plants have evolved a variety of enzymes, and non-enzyme antioxidant defense systems, that promote the removal of ROS and prevent oxidative damage to plant tissues. SOD and POD are well-studied antioxidant enzymes, and have been shown to help regulate oxidative reaction balance in resistance to $B$. cinerea. In our study, SOD and POD activities did not vary notably among the various lines at $0 \mathrm{~h}$ but increased as $B$. cinerea treatment time increased (Figures 3A,B). Similar results were obtained in studies of two apple cultivars (Bui et al., 2019). In our study, MDA content in all lines reached maximal value at $72 \mathrm{~h}$ (Figure 3C), possibly because the reduction of SOD and POD activities did not allow prompt relief of oxidative stress resulting from ROS accumulation. MDA levels in Col-0 and our gene silencing lines and overexpression lines were similar at later treatment times, as well as at $0 \mathrm{~h}$.

Increased ROS levels in response to external stimuli in plants led to a rise in melatonin levels and consequent activation of antioxidant enzyme activity (Liu et al., 2019). We observed changes in endogenous melatonin levels (Figure 2) resulting from up- or downregulation of ASMT and SNAT genes. Melatonin acts synergistically with antioxidant enzymes to remove ROS, promote photosynthesis, delay metabolite biosynthesis, enhance antioxidant capacity of plants, reduce oxidative stress in cells, tissues, or whole organisms, and protect plants from harsh environments (Manchester et al., 2015). Thus, increased endogenous melatonin promotes SOD and POD activity, facilitating prompt removal of excess ROS, and thereby enhancing plant defense against $B$. cinerea. Similarly, melatonin reduces damage to citrus fruits by penicillin fungal pathogens by removing ROS.

Various molecules secreted by $B$. cinerea induce the death of host cells (Siewers et al., 2005). Trypan blue staining revealed fewer dead cells in overexpression lines, and more dead cells

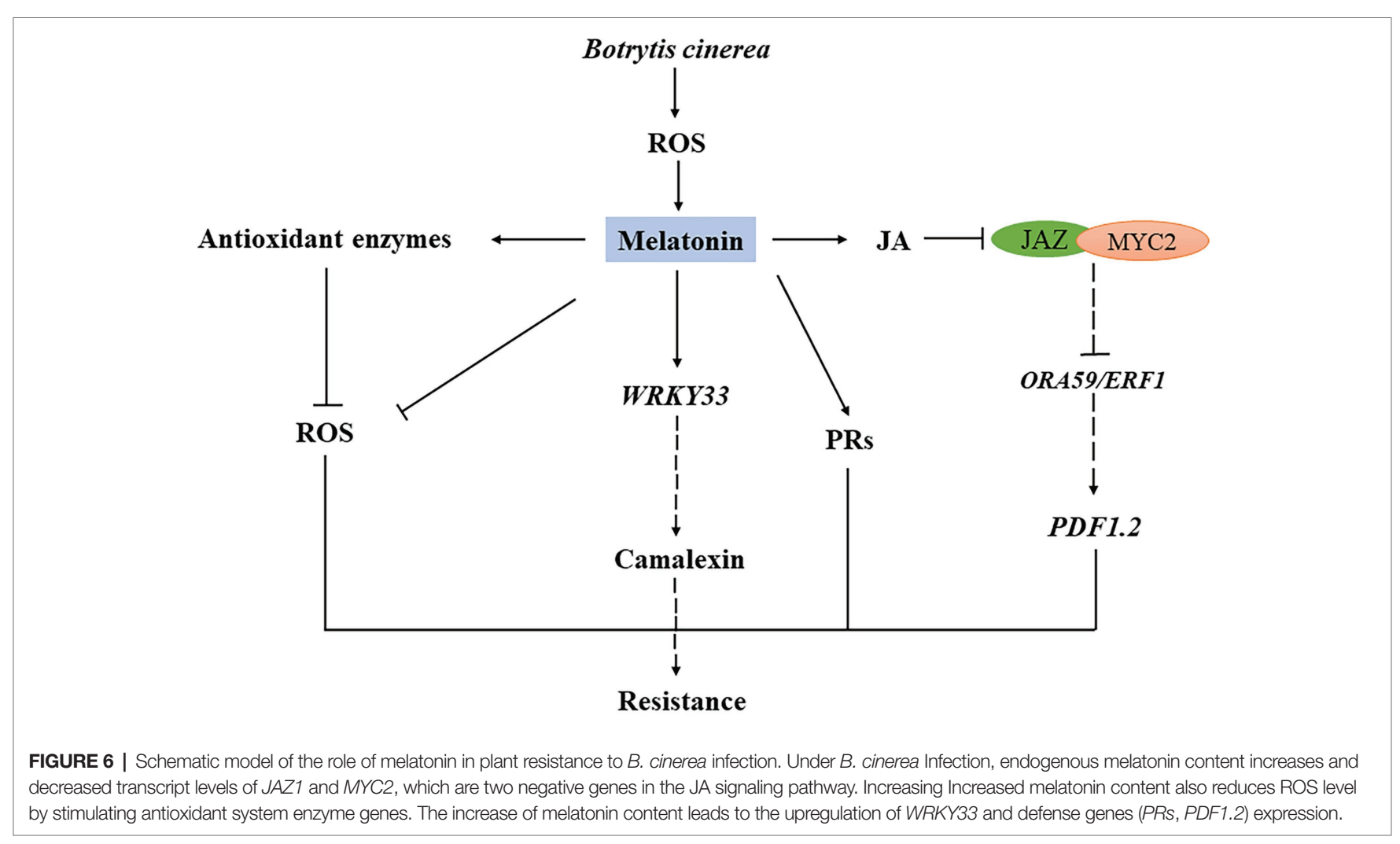


in gene silencing lines, relative to Col-0 (Figure 4A). A possible explanation is that necrotrophic stenosis induces ROS accumulation, triggering programmed cell death (PCD) in host cells and providing nutrients to fungi, thereby promoting their growth and reproduction, and the appearance of disease symptoms (Torres, 2010). Infection of leaves by B. cinerea results in obvious necrotic symptoms (Liu et al., 2017). Botrytis cinerea infection of apple plants similarly caused significant disease symptoms (Bui et al., 2019). Phenotypic observations of leaves in the present study revealed disease symptoms on 4 dai, and the appearance of large necrotic lesions by 6 dai (Figures 4B,C). Overexpression lines were less susceptible to $B$. cinerea relative to Col- 0 , whereas gene silencing lines were more susceptible and developed more obvious lesions (Figure 4D). Our findings suggest that ASMT and SNAT overexpression enhances plant resistance to $B$. cinerea by increasing melatonin level, consistently with previous reports that plant resistance to other fungi is enhanced by endogenous melatonin.

The growth of fungi was inhibited in a PDA medium containing melatonin, suggesting that melatonin enhances plant resistance (Arnao and Hernández-Ruiz, 2015). At various times $(24,48$, and $72 \mathrm{~h}$ ) following $B$. cinerea inoculation, the induced substrate for melatonin synthesis increases, and SNAT and ASMT overexpression leads to further melatonin synthesis (Figure 2), suggesting that melatonin is involved in resistance to $B$. cinerea. Plant infection with the pathogen Pseudomonas syringae pv. tomato DC3000 (Pst DC3000) similarly caused the increase of melatonin levels (Shi et al., 2015). Melatonin levels were high in our overexpression lines but lower in gene silencing lines. Melatonin content was maximal in ASMT-OE-1 at $48 \mathrm{~h}$, in ASMT-OE-2 at $24 \mathrm{~h}$, and in SNAT-OE-2 at $72 \mathrm{~h}$. This finding suggests that melatonin is being synthesized via multiple biosynthetic pathways.

In conclusion, we observed that increased endogenous melatonin level enhanced plant resistance to $B$. cinerea, consistently with previous reports. Melatonin is involved in the basic defensive responses of plants to $B$. cinerea and plays an essential role in plant immunity. Our findings provide new insights into molecular mechanisms of plant defensive signaling initiated by melatonin during interaction with pathogens, particularly fungal pathogens (Figure 6).

\section{REFERENCES}

AbuQamar, S., Moustafa, K., and Tran, L. S. (2017). Mechanisms and strategies of plant defense against Botrytis cinerea. Crit. Rev. Biotechnol. 37, 262-274. doi: 10.1080/07388551.2016.1271767

Aghdam, M. S., and Fard, J. R. (2017). Melatonin treatment attenuates postharvest decay and maintains nutritional quality of strawberry fruits (Fragariaxanannasa cv. Selva) by enhancing GABA shunt activity. Food Chem. 221, 1650-1657. doi: 10.1016/j.foodchem.2016.10.123

Amaral, F. G. D., and Cipolla-Neto, J. (2018). A brief review about melatonin, a pineal hormone. Arch. Endocrinol. Metab. 62, 472-479. doi: 10.20945/2359-3997000000066

An, C., and Mou, Z. (2013). The function of the mediator complex in plant immunity. Plant Signal. Behav. 8:e23182. doi: 10.4161/psb.23182

\section{DATA AVAILABILITY STATEMENT}

The original contributions presented in the study are included in the article/Supplementary Material, further inquiries can be directed to the corresponding authors.

\section{AUTHOR CONTRIBUTIONS}

The authors declare that the work described was original research that has not been published previously, and not under consideration for publication elsewhere, in whole or in part. All the authors listed have approved the manuscript that is enclosed. All authors contributed to the article and approved the submitted version.

\section{FUNDING}

This study was supported by grants from the National Natural Science Foundation of China (31300223), the Major Project of Basic Research Program of Natural Sciences of Shaanxi Province (2021JZ-41), the Natural Science Foundation of Shaanxi Province (2016JM3001), the Opening Foundation of Key Laboratory of Resource Biology and Biotechnology in Western China (Northwest University), the Ministry of Education, the First-class University and Academic programs of Northwest University, the Northwest University Graduate Innovation and Creativity Funds (YZZ17152), and the National Training Programs of Innovation and Entrepreneurship for Undergraduates (201910697021).

\section{ACKNOWLEDGMENTS}

The authors are grateful to Dr. S. Anderson for the English editing of the manuscript.

\section{SUPPLEMENTARY MATERIAL}

The Supplementary Material for this article can be found online at: https://www.frontiersin.org/articles/10.3389/fpls.2021.683228/ full\#supplementary-material

Arnao, M. B., and Hernández-Ruiz, J. (2015). Functions of melatonin in plants: a review. J. Pineal Res. 59, 133-150. doi: 10.1111/jpi.12253

Back, K., Tan, D. X., and Reiter, R. J. (2016). Melatonin biosynthesis in plants: multiple pathways catalyze tryptophan to melatonin in the cytoplasm or chloroplasts. J. Pineal Res. 61, 426-437. doi: 10.1111/jpi.12364

Bui, T. T. A., Wright, S. A. I., Falk, A. B., Vanwalleghem, T., and Davey, M. W. (2019). Botrytis cinerea differentially induces postharvest antioxidant responses in 'Braeburn' and 'Golden delicious' apple fruit. J. Sci. Food Agric. 99, 5662-5670. doi: 10.1002/jsfa.9827

Chen, J., Wang, H., Li, Y., Pan, J., Hu, Y., and Yu, D. (2018). Arabidopsis VQ10 interacts with WRKY8 to modulate basal defense against Botrytis cinerea. J. Integr. Plant Biol. 60, 956-969. doi: 10.1111/jipb.12664

Chen, Z., Xie, Y., Gu, Q., Zhao, G., Zhang, Y., Cui, W., et al. (2017). The AtrbohF-dependent regulation of ROS signaling is required for melatonin- 
induced salinity tolerance in Arabidopsis. Free Radic. Biol. Med. 108, 465-477. doi: 10.1016/j.freeradbiomed.2017.04.009

Dean, R., Van Kan, J. A., Pretorius, Z. A., Hammond-Kosack, K. E., Di Pietro, A., Spanu, P. D., et al. (2012). The top 10 fungal pathogens in molecular plant pathology. Mol. Plant Pathol. 13, 414-430. doi: 10.1111/j. 1364-3703.2011.00783.x

Dubbels, R., Reiter, R. J., Klenke, E., Goebel, A., Schnakenberg, E., Ehlers, C., et al. (1995). Melatonin in edible plants identified by radioimmunoassay and by high performance liquid chromatography-mass spectrometry. J. Pineal Res. 18, 28-31. doi: 10.1111/j.1600-079x.1995.tb00136.x

Fan, J., Xie, Y., Zhang, Z., and Chen, L. (2018). Melatonin: a multifunctional factor in plants. Int. J. Mol. Sci. 19:1528. doi: 10.3390/ijms19051528

Feng, B., and Shan, L. (2014). ROS open roads to roundworm infection. Sci. Signal. 7:pe10. doi: 10.1126/scisignal.2005273

Frey, M. E., D'Ippolito, S., Pepe, A., Daleo, G. R., and Guevara, M. G. (2018). Transgenic expression of plant-specific insert of potato aspartic proteases (StAP-PSI) confers enhanced resistance to Botrytis cinerea in Arabidopsis thaliana. Phytochemistry 149, 1-11. doi: 10.1016/j.phytochem. 2018.02.004

Ingle, R. A., Stoker, C., Stone, W., Adams, N., Smith, R., Grant, M., et al. (2015). Jasmonate signalling drives time-of-day differences in susceptibility of Arabidopsis to the fungal pathogen Botrytis cinerea. Plant J. 84, 937-948. doi: $10.1111 /$ tpj. 13050

Jones, J. D. G., and Dangl, J. L. (2006). The plant immune system. Nature 444, 323-329. doi: 10.1038/nature05286

Kang, K., Lee, K., Park, S., Byeon, Y., and Back, K. (2013). Molecular cloning of rice serotonin $\mathrm{N}$-acetyltransferase, the penultimate gene in plant melatonin biosynthesis. J. Pineal Res. 55, 7-13. doi: 10.1111/jpi.12011

Lerner, A. B., Case, J. D., Takahashi, Y., Lee, T. H., and Mori, W. (1958). Isolation of melatonin, the pineal gland factor that lightens melanocytes1. J. Am. Chem. Soc. 80:2587. doi: 10.1021/ja01543a060

Liu, C., Chen, L., Zhao, R., Li, R., Zhang, S., Yu, W., et al. (2019). Melatonin induces disease resistance to Botrytis cinerea in tomato fruit by activating jasmonic acid signaling pathway. J. Agric. Food Chem. 67, 6116-6124. doi: 10.1021/acs.jafc.9b00058

Liu, S., Ziegler, J., Zeier, J., Birkenbihl, R. P., and Somssich, I. E. (2017). Botrytis cinerea $\mathrm{B} 05.10$ promotes disease development in Arabidopsis by suppressing WRKY33-mediated host immunity. Plant Cell Environ. 40, 2189-2206. doi: 10.1111/pce.13022

López-Cruz, J., Crespo-Salvador, Ó., Fernández-Crespo, E., García-Agustín, P., and González-Bosch, C. (2016). Absence of cu-Zn-superoxide dismutase BCSOD1 reduces Botrytis cinerea virulence in Arabidopsis and in tomato plants, which reveals interplay among ROS, callose and signaling pathways. Mol. Plant Pathol. 18, 16-31. doi: 10.1111/mpp.12370

Manchester, L. C., Coto-Montes, A., Boga, J. A., Andersen, L. P., Zhou, Z., Galano, A., et al. (2015). Melatonin: an ancient molecule that makes oxygen metabolically tolerable. J. Pineal Res. 59, 403-419. doi: 10.1111/jpi.12267

Mandal, M. K., Suren, H., Ward, B., Boroujerdi, A., and Kousik, C. (2018). Differential roles of melatonin in plant-host resistance and pathogen suppression in cucurbits. J. Pineal Res. 65:e12505. doi: 10.1111/jpi.12505

Mao, G., Meng, X., Liu, Y., Zheng, Z., Chen, Z., and Zhang, S. (2011). Phosphorylation of a WRKY transcription factor by two pathogen-responsive MAPKs drives phytoalexin biosynthesis in Arabidopsis. Plant Cell 23, 1639-1653. doi: $10.1105 /$ tpc. 111.084996

Moustafa-Farag, M., and Almoneafy, A. (2019). Melatonin and its protective role against biotic stress impacts on plants. Biomol. Ther. 10:54. doi: 10.3390/ biom 10010054

Mulema, J. M., and Denby, K. J. (2012). Spatial and temporal transcriptomic analysis of the Arabidopsis thaliana-Botrytis cinerea interaction. Mol. Biol. Rep. 39, 4039-4049. doi: 10.1007/s11033-011-1185-4

Pardo-Hernández, M., López-Delacalle, M., and Rivero, R. M. (2020). ROS and NO regulation by melatonin under abiotic stress in plants. Antioxidants 9:1078. doi: $10.3390 /$ antiox9111078

Park, S., Byeon, Y., and Back, K. (2013). Functional analyses of three ASMT gene family members in rice plants. J. Pineal Res. 55, 409-415. doi: 10.1111/ jpi.12088

Pieterse, C. M., Leon-Reyes, A., Van der Ent, S., and Van Wees, S. C. (2009). Networking by small-molecule hormones in plant immunity. Nat. Chem. Biol. 5, 308-316. doi: 10.1038/nchembio. 164
Pingping, N., Xia, L., Shune, W., Jianhua, G., Hongwei, Z., and Dongdong, N. (2017). Induced systemic resistance against Botrytis cinerea by Bacillus cereus AR156 through a JA/ET- and NPR1-dependent signaling pathway and activates PAMP-triggered immunity in Arabidopsis. Front. Plant Sci. 8:238. doi: 10.3389/ fpls.2017.00238

Ramírez, V., Agorio, A., Coego, A., García-Andrade, J., Hernández, M. J., Balaguer, B., et al. (2011). MYB46 modulates disease susceptibility to Botrytis cinerea in Arabidopsis. Plant Physiol. 155, 1920-1935. doi: 10.1104/pp.110.171843

Ruan, J., Zhou, Y., Zhou, M., Yan, J., Khurshid, M., Weng, W., et al. (2019). Jasmonic acid signaling pathway in plants. Int. J. Mol. Sci. 20:2479. doi: $10.3390 /$ ijms 20102479

Schwab, R., Ossowski, S., Riester, M., Warthmann, N., and Weigel, D. (2006). Highly specific gene silencing by artificial microRNAs in Arabidopsis. Plant Cell 18, 1121-1133. doi: 10.1105/tpc.105.039834

Senthil-Kumar, M., and Mysore, K. S. (2013). Nonhost resistance against bacterial pathogens: retrospectives and prospects. Annu. Rev. Phytopathol. 51, 407-427. doi: $10.1146 /$ annurev-phyto-082712-102319

Shi, H., Chen, Y., Tan, D. X., Reiter, R. J., Chan, Z., and He, C. (2015). Melatonin induces nitric oxide and the potential mechanisms relate to innate immunity against bacterial pathogen infection in Arabidopsis. J. Pineal Res. 59, 102-108. doi: 10.1111/jpi.12244

Siewers, V., Viaud, M., Jimenez-Teja, D., Collado, I. G., Gronover, C. S., Pradier, J. M., et al. (2005). Functional analysis of the cytochrome P450 monooxygenase gene bcbot 1 of Botrytis cinerea indicates that botrydial is a strain-specific virulence factor. Mol. Plant-Microbe Interact. 18, 602-612. doi: 10.1094/mpmi-18-0602

Sun, C., Liu, L., Wang, L., Li, B., Jin, C., and Lin, X. (2020). Melatonin: a master regulator of plant development and stress responses. J. Integr. Plant Biol. 63, 126-145. doi: 10.1111/jipb.12993

Torres, M. A. (2010). ROS in biotic interactions. Physiol. Plant. 138, 414-429. doi: 10.1111/j.1399-3054.2009.01326.x

Wang, C., Ding, Y., Yao, J., Zhang, Y., and Mou, Z. (2015). Arabidopsis Elongator subunit 2 positively contributes to resistance to the necrotrophic fungal pathogens Botrytis cinerea and Alternaria brassicicola. Plant J. 83, 1019-1033. doi: $10.1111 /$ tpj. 12946

Wang, J., Xia, X. M., Wang, H. Y., Li, P. P., and Wang, K. Y. (2013). Inhibitory effect of lactoferrin against gray mould on tomato plants caused by Botrytis cinerea and possible mechanisms of action. Int. J. Food Microbiol. 161, 151-157. doi: 10.1016/j.ijfoodmicro.2012.11.025

Wei, Y., Hu, W., Wang, Q., Zeng, H., Li, X., Yan, Y., et al. (2017). Identification, transcriptional and functional analysis of heat-shock protein 90s in banana (Musa acuminata L.) highlight their novel role in melatonin-mediated plant response to Fusarium wilt. J. Pineal Res. 62:e12367. doi: 10.1111/jpi.12367

Williamson, B., Tudzynski, B., Tudzynski, P., and Van Kan, J. A. L. (2007). Botrytis cinerea: the cause of grey mould disease - WILLIAMSON - 2007 molecular plant pathology - Wiley online library. Mol. Plant Pathol. 8, 561-580. doi: 10.1111/j.1364-3703.2007.00417.x

Yao, J. W., Ma, Z., Ma, Y. Q., Zhu, Y., Lei, M. Q., Hao, C. Y., et al. (2020). Role of melatonin in UV-B signalling pathway and UV-B stress resistance in Arabidopsis thaliana. Plant Cell Environ. 44, 114-129. doi: 10.1111/pce.13879

Yin, L., Wang, P., Li, M., Ke, X., Li, C., Liang, D., et al. (2013). Exogenous melatonin improves Malus resistance to Marssonina apple blotch. J. Pineal Res. 54, 426-434. doi: 10.1111/jpi.12038

Zang, D., Wang, C., Ji, X., and Wang, Y. (2015). Tamarix hispida zinc finger protein ThZFP1 participates in salt and osmotic stress tolerance by increasing proline content and SOD and POD activities. Plant Sci. 235, 111-121. doi: 10.1016/j.plantsci.2015.02.016

Zhang, S., Zheng, X., Reiter, R. J., Feng, S., Ying, W., Liu, S., et al. (2017). Melatonin attenuates potato late blight by disrupting cell growth, stress tolerance, fungicide susceptibility and homeostasis of gene expression in Phytophthora infestans. Front. Plant Sci. 8:1993. doi: 10.3389/fpls.2017. 01993

Zhao, D., Wang, H., Chen, S., Yu, D., and Reiter, R. J. (2021). Phytomelatonin: an emerging regulator of plant biotic stress resistance. Trends Plant Sci. 26, 70-82. doi: 10.1016/j.tplants.2020.08.009

Zheng, Y., Yang, Y., Liu, C., Chen, L., Sheng, J., and Shen, L. (2015). Inhibition of SIMPK1, SlMPK2, and SIMPK3 disrupts defense signaling pathways and enhances tomato fruit susceptibility to Botrytis cinerea. J. Agric. Food Chem. 63, 5509-5517. doi: 10.1021/acs.jafc.5b00437 
Zhu, Y., Gao, H., Lu, M., Hao, C., Pu, Z., Guo, M., et al. (2019). Melatoninnitric oxide crosstalk and their roles in the redox network in plants. Int. J. Mol. Sci. 20:6200. doi: 10.3390/ijms20246200

Conflict of Interest: The authors declare that the research was conducted in the absence of any commercial or financial relationships that could be construed as a potential conflict of interest.
Copyright (c) 2021 Zhu, Guo, Song, Zhang, Guo, Hou, Hao, An and Huang. This is an open-access article distributed under the terms of the Creative Commons Attribution License (CC BY). The use, distribution or reproduction in other forums is permitted, provided the original author(s) and the copyright owner(s) are credited and that the original publication in this journal is cited, in accordance with accepted academic practice. No use, distribution or reproduction is permitted which does not comply with these terms. 